\title{
A Finite de Finetti Theorem for Infinite-Dimensional Systems
}

\author{
Christian D'Cruz $*$ Tobias J. Osborne, and Rüdiger Schack \\ Department of Mathematics, Royal Holloway, University of London, UK
}

(Dated: August 11, 2018)

\begin{abstract}
We formulate and prove a de Finetti representation theorem for finitely exchangeable states of a quantum system consisting of $k$ infinite-dimensional subsystems. The theorem is valid for states that can be written as the partial trace of a pure state $|\Psi\rangle\langle\Psi|$ chosen from a family of subsets $\left\{\mathcal{C}_{n}\right\}$ of the full symmetric subspace for $n$ subsystems. We show that such states become arbitrarily close to mixtures of pure power states as $n$ increases. We give a second equivalent characterization of the family $\left\{\mathcal{C}_{n}\right\}$.
\end{abstract}

The classical de Finetti theorem [1, 2] is a representation theorem for exchangeable probability distributions. It is of fundamental importance for the analysis of repeated trials in Bayesian statistics [3]. For positive integers $n$ and $k$, a joint probability distribution for $k$ random variables is said to be $n$-exchangeable, or simply finitely exchangeable, if it can be written as the marginal of a symmetric distribution for $n$ variables. A distribution is said to be infinitely exchangeable if it is $n$-exchangeable for all $n$. The content of the de Finetti theorem is that any infinitely exchangeable probability distribution can be written as a convex mixture of power distributions [1, 4]. Additionally, finitely exchangeable distributions can be approximated by such mixtures [2].

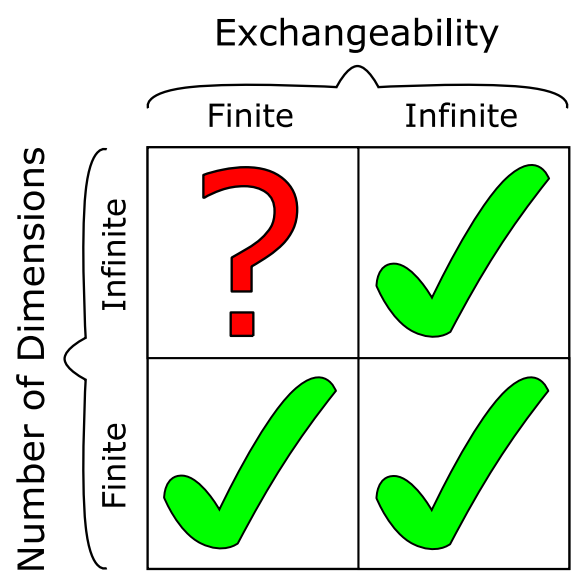

FIG. 1: Quantum generalizations of the classical de Finetti theorem fall into four classes. One can assume finite or infinite exchangeability, and the subsystems can be finite or infinite-dimensional. In the case of infinite exchangeability, we study a state $\rho_{k}$ that can be written as $\rho_{k}=\operatorname{tr}_{n-k}\left(\rho_{n}\right)$, for every integer $n>k$ for a symmetric state $\rho_{n}$. The theorems then state that $\rho_{k}$ can be written as a mixture of power states [5, 6, 7]. If finite exchangeability is assumed, the quantum de Finetti theorem says that a state $\rho_{k}=\operatorname{tr}_{n-k}\left(\rho_{n}\right)$, for a fixed value of $n$ and symmetric state $\rho_{n}$, can be approximated by a mixture of power states. The remaining case of finite exchangeability and infinite-dimensional subsystems is the topic of this Letter.

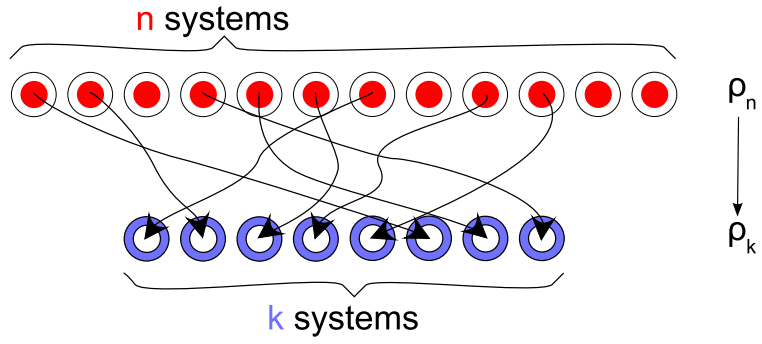

FIG. 2: A constructive illustration of symmetric states. A state $\rho_{n}$ is symmetric when $\pi \rho_{n} \pi^{\dagger}=\rho_{n}$ for all permutations $\pi$. In the figure, we illustrate these states as those for which the state $\rho_{k}$ is independent of the choice of $n-k$ subsystems to trace out, and of the order in which we place the remaining $k$.

In recent years there has been increased interest in quantum analogues of the de Finetti theorem. Figure 1 gives an overview of the possibilities. They are of fundamental interest in mathematics [6, 8, 9], quantum information theory 7, 10, 11, 12], and quantum foundations [7]. Concrete applications include quantum state tomography [7, 13], quantum process tomography [14], entanglement purification [11], and quantum cryptography [15, 16]. Despite the progress in this field, it remains an open question what quantum de Finetti theorems exist for finitely exchangeable states on an array of infinitedimensional subsystems. A direct generalization of the classical theorem to all finitely exchangeable quantum states is impossible due to a counterexample given in [12]. There the authors construct, for any integer $n>2$, an $n$-exchangeable state on two infinite-dimensional subsystems that has a trace distance of at least $1 / 2$ from any mixture of power states [5].

In this Letter we prove a quantum de Finetti theorem for a particular class of $n$-exchangeable quantum states on a Hilbert space $\mathcal{H}^{\otimes k}$, where $\mathcal{H}$ is infinite-dimensional, and where $n$ and $k$ are arbitrary. Our class consists of all those pure states $\rho_{n}=|\psi\rangle\langle\psi|$ where $|\psi\rangle$ can be written as a superposition of the form $\int d \gamma c_{\gamma}|\gamma\rangle^{\otimes n}$ and each $|\gamma\rangle$ is a coherent state. It should be noted that such a superposition is very different from a mixture of 
power states. This class contains many physically relevant states, the simplest example being the Schrödinger cat states $|\alpha\rangle^{\otimes n}+|\beta\rangle^{\otimes n}$ of $n$ optical modes. While such states are covered by the finite de Finetti theorem, in reality they are often an approximation to a continuous superposition $\int d \gamma c_{\gamma}|\gamma\rangle^{\otimes n}$ where $c_{\gamma}$ is strongly peaked around $\alpha$ and $\beta$. Such states can only be incorporated by our infinite version of the quantum de Finetti theorem, since they cannot be represented on any tensor product space $\mathcal{V}^{\otimes n}$, where $\mathcal{V}$ is a finite-dimensional subspace of $\mathcal{H}$. We note that several current experiments dealing with systems of many identical particles [17, 18] can produce continuous cat states of the form $\int d \gamma c_{\gamma}|\gamma\rangle^{\otimes n}$. Some examples include double-well Bose-Einstein condensates [19], superconducting current loops 20, 21], and spinpolarised atomic ensembles 22].

The Letter is structured as follows. Having defined in more detail the family of subsets $\left\{\mathcal{C}_{n}\right\}$ to which our theorem applies, we state our theorem and give an outline of the steps involved in proving it. The latter parts consider the details of the proof, and we conclude with some remarks about the extension of this theorem to larger symmetric subspaces.

Coherent States. - We begin by introducing the states which we shall study. Consider a finite collection of $n$ quantum systems each with a countably infinite Hilbert space $\mathcal{H}$, such as $n$ modes of the electromagnetic field. Let $|0\rangle,|1\rangle, \ldots$ label an arbitrary basis for $\mathcal{H}$. A tensorproduct basis state $|\boldsymbol{x}\rangle$ of $\mathcal{H}^{\otimes n}$, labelled by the word $\boldsymbol{x}$, is given by $|\boldsymbol{x}\rangle=\left|x_{1}\right\rangle \otimes \ldots \otimes\left|x_{n}\right\rangle$. Let the annihilation operator $a$ be the operator that lowers the basis states according to $a|j\rangle=\sqrt{j}|j-1\rangle$, generalizing the standard definition for harmonic oscillators. For $\alpha \in \mathbb{C}$, a coherent state $|\alpha\rangle$ is the eigenstate of the annihilation operator with eigenvalue $\alpha$ [23]. In this Letter, we prove a de Finetti theorem for the span $\mathcal{C}_{n}$ of coherent power states $|\alpha\rangle^{\otimes n}$.

An equivalent description of $\mathcal{C}_{n}$. - The above definition of $\mathcal{C}_{n}$ is in terms of the overcomplete spanning set $\left\{|\alpha\rangle^{\otimes n} \mid \alpha \in \mathbb{C}\right\}$, and so does little to elucidate the states that it encompasses. We shall provide a brief alternative definition in terms of a basis which is closely linked to the multinomial basis for the symmetric subspace.

The space $\mathcal{S}_{n}$ is defined to be the span of states $|w\rangle$, given by

$$
|w\rangle=\sqrt{\frac{1}{n^{w}}}\left(\sum_{\boldsymbol{y}, \sum y_{i}=w} \sqrt{\frac{w !}{y_{1} ! \ldots y_{n} !}}|\boldsymbol{y}\rangle\right),
$$

for all $w \in \mathbb{Z}, w \geq 0$. It is possible to show that $\mathcal{S}_{n} \equiv \mathcal{C}_{n}$ [24], and so the states $\{|w\rangle\}$ provide an orthonormal countable basis of the coherent power subspace. They are analogous to the classical urn model of $w$ tosses of a fair $n$-sided coin.

This second characterization allows us to gain an insight into the relative size of $C_{n}$ and the full symmetric subspace, Sym $\mathcal{H}^{\otimes n}$. To do so, we define the $w$-weighted subspace to be the symmetric span of all states $|\boldsymbol{x}\rangle$ that satisfy $\sum x_{i}=w$ and we note that $\operatorname{Sym} \mathcal{H}^{\otimes n}$ is the direct sum of these. The dimension of the $w$-weighted subspaces grows polynomially with $w$, but in contrast $C_{n}$ contains only the state $|w\rangle$ with weight $w$.

$A$ de Finetti theorem.- - If $\rho_{n}$ is a pure state on a particular $\mathcal{C}_{n}$, we are able to approximate the reduction to $k$ systems $\operatorname{tr}_{n-k}\left(\rho_{n}\right)$ as a mixture of coherent power state projectors. More precisely, we can construct a probability measure $\nu(\alpha)$ such that

$$
\Delta \equiv\left\|\operatorname{tr}_{n-k}\left(\rho_{n}\right)-\int(|\alpha\rangle\langle\alpha|)^{\otimes k} \nu(\alpha) d^{2} \alpha\right\|_{1} \leq \frac{3}{2} \frac{k}{n} .
$$

Here, the trace norm $\|\sigma\|_{1}$ is the sum of the absolute values of the eigenvalues of $\sigma$.

Proof Outline.- To prove this theorem, two main steps are necessary. We begin by constructing the operator

$$
\Lambda_{n, k}=\frac{n-k}{\pi} \int d^{2} \alpha I_{k} \otimes(|\alpha\rangle\langle\alpha|)^{\otimes n-k},
$$

where $I_{k}$ is the identity on the first $k$ subsystems. We show that its restriction to the $\operatorname{span} \mathcal{C}_{n}$ of coherent power states is equal to the identity, which transforms the term $\operatorname{tr}_{n-k}\left(\rho_{n}\right)$, in Eq. (2), into an integral. This allows the use of standard inequalities to bound $\Delta$ from above.

The displacement operator, defined below, plays the rôle of the unitary transformations from [12]. The crucial fact is that in our integral $\Lambda_{n, k}$, analogous to that used in [12], we have no divergent dimension-dependent factor, which would be the case for a direct application of the existing proof. Without this an infinite-dimensional result is not possible.

An identity operator for coherent power states.- We now wish to show that $\Lambda_{n, k}$ is the identity operator on $\mathcal{C}_{n}$. In order to study the multi-mode states, we note that the single-mode states have an explicit expansion in the basis $|0\rangle,|1\rangle, \ldots$

$$
|\alpha\rangle=e^{-|\alpha|^{2} / 2} \sum_{i=0}^{\infty} \frac{\alpha^{i}}{\sqrt{i !}}|i\rangle=D(\alpha)|0\rangle,
$$

where $D(\alpha)$ is the displacement operator, $D(\alpha)=$ $e^{\alpha a^{\dagger}-\bar{\alpha} a}$.

The proof takes two parts. We first show that $\Lambda_{n, k}$ acts identically upon the vacuum state, and move on to show that it commutes with the displacement operator. Explicitly we intend to show

$$
\begin{aligned}
\Lambda_{n, k}|\mathbf{0}\rangle & =|\mathbf{0}\rangle, \\
{\left[\Lambda_{n, k}, D(\alpha)^{\otimes n}\right] } & =0 .
\end{aligned}
$$

We prove Eq. (5) by using the expansion of a coherent state given in Eq. (4). Consider the inner product of 
$\Lambda_{n, k}|\mathbf{0}\rangle$ with a basis vector labelled by a word $\boldsymbol{x}$.

$$
\begin{aligned}
& \left\langle\boldsymbol{x}\left|\Lambda_{n, k}\right| \mathbf{0}\right\rangle= \\
& \quad \frac{n-k}{\pi} \delta_{x_{1}, 0} \ldots \delta_{x_{k}, 0} \int d^{2} \alpha e^{-(n-k)|\alpha|^{2}} \prod_{i=k+1}^{n} \frac{\bar{\alpha}^{x_{i}}}{\sqrt{x_{i} !}} .
\end{aligned}
$$

The integral is zero unless $x_{i}=0$ for $k<i \leq n$, in which case we obtain

$$
\left\langle\boldsymbol{x}\left|\Lambda_{n, k}\right| \mathbf{0}\right\rangle= \begin{cases}1 & \text { if } \boldsymbol{x}=\mathbf{0} \\ 0 & \text { otherwise }\end{cases}
$$

This means that $\Lambda_{n, k}|\mathbf{0}\rangle=|\mathbf{0}\rangle$. While proving Eq. (6) we employ the notation $D_{\alpha}^{m}=D(\alpha)^{\otimes m}$.

$$
\begin{aligned}
\Lambda_{n, k} & D_{\alpha}^{n}=\frac{n-k}{\pi} \int d^{2} \beta I_{k} \otimes\left(D_{\beta}^{n-k}|\mathbf{0}\rangle\langle\mathbf{0}| D_{-\beta}^{n-k}\right) D_{\alpha}^{n} \\
& =\frac{n-k}{\pi} \int d^{2} \beta D_{\alpha}^{k} \otimes\left(D_{\beta}^{n-k}|\mathbf{0}\rangle\langle\mathbf{0}|\left[D_{-\beta}^{n-k} D_{\alpha}^{n-k}\right]\right) \\
& =\frac{n-k}{\pi} \int d^{2} \gamma D_{\alpha}^{k} \otimes\left(\left[D_{\alpha}^{n-k} D_{\gamma}^{n-k}\right]|\mathbf{0}\rangle\langle\mathbf{0}| D_{-\gamma}^{n-k}\right) \\
& =\frac{n-k}{\pi} \int d^{2} \gamma D_{\alpha}^{n}\left(I_{k} \otimes D_{\gamma}^{n-k}|\mathbf{0}\rangle\langle\mathbf{0}| D_{-\gamma}^{n-k}\right) \\
& =D_{\alpha}^{n} \Lambda_{n, k}=D(\alpha)^{\otimes n} \Lambda_{n, k},
\end{aligned}
$$

where we have substituted $\gamma=\beta-\alpha$ and used the property $D(x+y)=e^{\frac{1}{2}(x \bar{y}-\bar{x} y)} D(x) D(y)$.

To complete the proof we combine the two previous results,

$$
\begin{aligned}
\Lambda_{n, k}|\alpha\rangle^{\otimes n} & =\Lambda_{n, k} D(\alpha)^{\otimes n}|\mathbf{0}\rangle \\
& =D(\alpha)^{\otimes n} \Lambda_{n, k}|\mathbf{0}\rangle \\
& =D(\alpha)^{\otimes n}|\mathbf{0}\rangle \\
& =|\alpha\rangle^{\otimes n}
\end{aligned}
$$

Establishing the bound on $\Delta$.- Having demonstrated that we have a resolution of the identity on $\mathcal{C}_{n}$ we can now begin the proof of the theorem itself. The state $\rho_{n}$ is pure, and thus is given by some $\rho_{n}=|\Psi\rangle\langle\Psi|$. For each $\alpha$ we define a non-normalized state on the first $k$ subsystems

$$
\left|\Psi_{k}^{\alpha}\right\rangle=\sqrt{\frac{n-k}{\pi}}\left(I_{k} \otimes\left\langle\left.\alpha\right|^{\otimes n-k}\right)|\Psi\rangle,\right.
$$

with corresponding positive operator

$$
\begin{aligned}
\rho_{k}^{\alpha} & =\left|\Psi_{k}^{\alpha}\right\rangle\left\langle\Psi_{k}^{\alpha}\right| \\
& =\frac{n-k}{\pi} \operatorname{tr}_{n-k}\left(I_{k} \otimes(|\alpha\rangle\langle\alpha|)^{\otimes n-k}|\Psi\rangle\langle\Psi|\right) .
\end{aligned}
$$

Since $|\Psi\rangle \in \mathcal{C}_{n}$, we note that

$$
\int \rho_{k}^{\alpha} d^{2} \alpha=\operatorname{tr}_{n-k}\left(\Lambda_{n, k}|\Psi\rangle\langle\Psi|\right)=\operatorname{tr}_{n-k}\left(\rho_{n}\right) .
$$

To define the measure $\nu(\alpha)$ in Eq. (2), we further project the states $\rho_{k}^{\alpha}$ onto $P^{\alpha}=|\alpha\rangle\left\langle\left.\alpha\right|^{\otimes k}\right.$ and define $\nu(\alpha)=\operatorname{tr}\left(P^{\alpha} \rho_{k}^{\alpha}\right)$, so that $|\alpha\rangle\left\langle\left.\alpha\right|^{\otimes k} \nu(\alpha)=P^{\alpha} \rho_{k}^{\alpha} P^{\alpha}\right.$. We then have

$$
\begin{aligned}
\Delta & =\left\|\int d^{2} \alpha\left(\rho_{k}^{\alpha}-P^{\alpha} \rho_{k}^{\alpha} P^{\alpha}\right)\right\|_{1} \\
\leq & \underbrace{\left\|\int d^{2} \alpha\left(\rho_{k}^{\alpha}-P^{\alpha} \rho_{k}^{\alpha}\right)\right\|_{1}}_{\zeta} \\
& +\underbrace{\left\|\int d^{2} \alpha\left(\rho_{k}^{\alpha}-\rho_{k}^{\alpha} P^{\alpha}\right)\right\|_{1}}_{\eta} \\
& +\underbrace{\left\|\int d^{2} \alpha\left(I_{k}-P^{\alpha}\right) \rho_{k}^{\alpha}\left(I_{k}-P^{\alpha}\right)\right\|_{1}}_{\theta},
\end{aligned}
$$

using an identity presented in [12]-

$$
A-B A B=(A-B A)+(A-A B)-(I-B) A(I-B) \text {. }
$$

And so it is necessary to calculate bounds for $\zeta, \eta$ and $\theta$. We may do so by employing the completeness relation that $\Lambda_{n, k}$ provides.

$$
\begin{aligned}
\zeta & =\left\|\operatorname{tr}_{n-k}\left(\rho_{n}\right)-\int d^{2} \alpha \frac{n-k}{\pi} \operatorname{tr}_{n-k}\left(|\alpha\rangle\left\langle\left.\alpha\right|^{\otimes n} \rho_{n}\right) \|_{1}\right.\right. \\
& =\left\|\operatorname{tr}_{n-k}\left(\rho_{n}\right)-\frac{n-k}{n} \operatorname{tr}_{n-k}\left(\Lambda_{n, 0} \rho_{n}\right)\right\| \|_{1} \\
& =\left(1-\frac{n-k}{n}\right)\left\|\operatorname{tr}_{n-k}\left(\rho_{n}\right)\right\|_{1}=\frac{1}{2} \frac{k}{n}
\end{aligned}
$$

Similarly, we have that $\eta=\frac{1}{2} \frac{k}{n}$. Bounding $\theta$ is only marginally more complicated. Beginning with the triangle inequality,

$$
\begin{aligned}
\theta & \leq \int d^{2} \alpha\left\|\left(I_{k}-P^{\alpha}\right) \rho_{n}^{\alpha}\left(I_{k}-P^{\alpha}\right)\right\|_{1} \\
& \leq \frac{1}{2} \int d^{2} \alpha \operatorname{tr}\left|\left(I_{k}-P^{\alpha}\right) \rho_{n}^{\alpha}\left(I_{k}-P^{\alpha}\right)\right|
\end{aligned}
$$

Now, since we have the projector, $I_{k}-P^{\alpha}$, straddling a completely positive operator, $\rho_{n}^{\alpha}$, this simplifies to

$$
\begin{aligned}
\theta & \leq \frac{1}{2} \int d^{2} \alpha \operatorname{tr}\left(\left(I_{k}-P^{\alpha}\right) \rho_{n}^{\alpha}\right) \\
& \leq \frac{1}{2} \operatorname{tr}\left(\int d^{2} \alpha\left(I_{k}-P^{\alpha}\right) \rho_{n}^{\alpha}\right) \\
& \leq \frac{1}{2} \operatorname{tr}\left(\left(1-\frac{n-k}{n}\right) \operatorname{tr}_{n-k} \rho_{n}\right)=\frac{1}{2} \frac{k}{n}
\end{aligned}
$$

Bringing this all together gives us the final bound since $\Delta \leq \zeta+\eta+\theta$. 
Conclusion.- We have stated and proved a de Finetti theorem for a limited class of finitely exchangeable states in a Hilbert space of countably infinite dimension. The counterexample given in 12] shows that we shall never have a direct generalisation of the classical scenario. An important question concerns the characterization of the set of finitely exchangeable states for which approximate de Finetti representations do exist. Our work provides a partial answer to this question for a class of states to which the previously known de Finetti theorems do not apply.

It is possible to extend our results to a larger class of states by considering the countably infinite Hilbert space $\mathcal{H}$ as a tensor product of two, or possibly more, subsystems. For example, $\mathcal{H}$ is equivalent to a tensor product of itself and a qubit, providing both a new family of de Finetti states and a new bound on $\Delta$. This is the subject of ongoing work.

This work was supported by the UK Engineering and Physical Sciences Research Council. We thank Renato Renner, Robert König, and Graeme Mitchison for useful discussions.

* Electronic address: C.H.D-Cruz@rhul.ac.uk

[1] B. de Finetti, Ann. Inst. H. Poincaré 7, 1 (1937).

[2] P. Diaconis and D. Freedman, Ann. Prob. 8, 745 (1980).

[3] L. J. Savage, The Foundations of Statistics (Dover, New York, 1972), 2nd ed.

[4] A power distribution $Y$ of $n$ variables is a probability density function given by $Y\left(x_{1}, x_{2}, \ldots, x_{n}\right)=$ $X\left(x_{1}\right) X\left(x_{2}\right) \ldots X\left(x_{n}\right)$ where $X$ is a single-variable density function.

[5] A power state on an $n$-mode system is one that may be written in the form $\sigma^{\otimes n}$, where $\sigma$ is a single-mode density operator.
[6] R. L. Hudson and G. R. Moody, Z. Wahrschein. verw. Geb. 33, 343 (1976).

[7] C. M. Caves, C. A. Fuchs, and R. Schack, J. Math. Phys. 43, 4537 (2002), quant-ph/0104088.

[8] E. Størmer, J. Funct. Anal 3, 48 (1969).

[9] D. Petz, Prob. Th. Rel. Fields 85, 1 (1990).

[10] R. König and R. Renner, J. Math. Phys 46, 122108 (2005), quant-ph/0410229.

[11] T. A. Brun, C. M. Caves, and R. Schack, Phys. Rev. A 63, 042309 (2001), quant-ph/0010038

[12] M. Christandl, R. König, G. Mitchison, and R. Renner (2006), quant-ph/0602130.

[13] R. Schack and C. A. Fuchs, in Quantum State Estimation, edited by M. G. A. Paris and J. Řeháček (SpringerVerlag, Berlin, 2004), p. 147, quant-ph/0404156

[14] C. A. Fuchs, R. Schack, and P. F. Scudo, Phys. Rev. A 69, 062305 (2004), quant-ph/0307198.

[15] R. Renner, Ph.D. thesis, Zurich (2005), quant-ph/0512258.

[16] R. Renner, N. Gisin, and B. Kraus, Phys. Rev. A 72 , 012332 (2005), quant-ph/0502064.

[17] J. I. Korsbakken, K. B. Whaley, J. DuBois, and J. I. Cirac (2006), quant-ph/0611121.

[18] These systems can be modelled as a collection of $n$ harmonic oscillator modes.

[19] N. R. Claussen, E. A. Donley, S. T. Thompson, and C. E. Wieman, Nature 417, 529 (2002).

[20] C. H. van der Wal, A. C. J. ter Haar, F. K. Wilhelm, R. N. Schouten, C. J. P. M. Harmans, T. P. Orlando, S. Lloyd, and J. E. Mooij, Science 290, 773 (2000).

[21] J. R. Friedman, V. Patel, W. Chen, S. K. Tolpygo, and J. E. Lukens, Nature 406, 43 (2000).

[22] B. Julsgaard, A. Kozhekin, and E. S. Polzik, Nature 413, 400 (2001).

[23] W. H. Louisell, Quantum Statistical Properties of Radiation (Wiley, New York, 1990).

[24] To prove this we use the projector $\Lambda_{n, 0}$ defined in Eq. (3). This allows us to write $|w\rangle=\int d^{2} \beta w_{\beta}|\beta\rangle^{\otimes n}$ and $|\alpha\rangle^{\otimes n}=\sum \alpha_{w^{\prime}}\left|w^{\prime}\right\rangle$ for all $\alpha$ and $w$, giving $\mathcal{S}_{n} \subset \mathcal{C}_{n} \subset \mathcal{S}_{n}$. 